\title{
Article \\ Matrix Product State Simulations of Non-equilibirum Steady States and Transient Heat Flows in the Two-Bath Spin-Boson Model at Finite Temperatures
}

\author{
Angus J Dunnett ${ }^{1} *$ and Alex W Chin ${ }^{1}$, \\ 1 Insitut des Nanosciences de Paris, Sorbonne Universit, CNRS, Place Jussieu,Paris, 75005 \\ * Correspondence: angus.dunnett@insp.upmc.fr
}

\begin{abstract}
Non-perturbative, polarons, non-eqilibrium,etc.,etc.
Keywords: Open quantum systems, Tensor networks, non-equilibrium dynamics
\end{abstract}

\section{Introduction}

The physics of open quantum systems (OQS) play a critical role in almost all aspects of quantum science[1,2], and the emergent phenomena of dephasing, decoherence and dissipation particularly limit our ability to initialise and control multi-partite quantum states. As a direct result of this, the development of scalable quantum technologies is greatly contrained by open system phenomena, and understanding how irreversibility arises from microscopic system-environment interactions has become essential for finding ways to mitigate deleterious noise efects [3]. However, alongside this goal of suppressing dissipative noise - normally by making the systems less 'open' - the theory of OQS also plays a vital role in the design of systems where the exploitation of strong energy and information exchange between a system and its environment is desirable: this is the world of quantum thermodynamics and nanoscale energy harvesting, storage and transduction [4-6].

Any 'machine' or device capable of converting ambient energy into work must necessarily be an open system. As these machines shrink to lengths where such energetic transformations can become few-quanta, ultrafast events, it becomes necessary to describe their functional dynamics on timescales over which system-environment correlations - in both space and time - may be highly relevant $[7,8]$. Unlike the perturbative OQS found, for example, in atomic systems where dissipation can be characterised by simple decay rates, quantum energy harvesting naturally focuses on the highly non-markovian and non-perturbative regime of OQS where the border between the 'system' and 'environmental' degrees of freedoms is ill-defined. Moreover, as systems capable of converting thermal energy must also reject a certain amount of heat to a colder reservoir [6], the study of quantum energy harvesting leads directly to a consideration of multi-environment OQS, and the extended, inter-environmental quantum correlations that could be generated under non-equilibrium operating conditons.

Molecular and biological light-harvesting systems provide a good example of such nanoscale energy extraction, in which a non-thermal population of electronic excitations ( excitons, charge pairs...) appears from the molecule-mediated connection of the 'hot' photon and 'cold' vibrational environments. In this context, much attention has been placed on the complex physics due to the strong coupling and non-separable timescales of electronic and environmental (vibrational) dynamics [9-11], which include potentially exploitable effects such as transient breaking of detailed balance [12], noise-induced electronic coherence and cooperative multi-environment effects $[13,14]$. In such studies, the effect of light is normally assumed to be weak, leading to the 'additive' approximation that 
phenomenological terms describing excitation, emission and dephasing can be simply added to the more complex equations of motions of the vibronic open system. However, organic molecules often have very strong light-matter coupling and can show surprising non-additive effects $[15,16]$, including nonlinear polaritonic weakening of exciton-phonon coupling in microcavity systems [17].

The example, above, highlights the theoretical challenges posed by some energy harvesting systems: non-perturbative and highly structured couplings, comparable dynamical timescales and competiting environmental processes. Under these conditions the dissipative dynamics of the system's reduced density matrix cannot be simply described by dephasing and relaxation rates: the full real-time evolution of the system and its environments must be accounted for an essentially equal footing. This looks, a priori, like a hopeless task, as each environment contains a continuum of quantum excitation modes, and the formal number of quantum states in any computation will explode exponentially in the number of such modes. However, things are not so desperate, and two broad responses to this problem have emerged over recent years: one branch aims to efficiently simulate the propagators of the system's reduced density matrix [9,18-20], the other aims at representing and evolving the entire system-environment wave function. Important contributions in this latter domain are the DMRG-based TEDOPA technique [21,22], Dissipation-Assisted Matrix Product Factorization [23], Time-Dependent Numerical Renormalisation Group techniques and the Multi-Layer Multi Configurational Time-Dependent Hartree method (ML-MCTDH) developed in Chemical physics $[24,25]$.

The key to all of the wave function methods is the observation that, given a well-defined initial condition, the quantum dyanmics generated by typical system-environment Hamiltonians leave the state inside a much smaller sub-space of the complete Hilbert space of the problem. This suggests that the wave function can be parametrised by a potentially tractable number of parametres, and - as we shall see - the effectively short-range, one-dimensional structure of OQS Hamiltonians implies that Matrix Product States (MPS) will provide an efficient and versatile format for many system-environment wave functions. Viewed this way, the parameters (matrics) of an MPS can be consdiered as variational degrees of freedom, leading to the powerful 1-site time-dependent varitional principle (1TDVP) algorithm for efficient propagation of large wave functions in real-time [26]. This general technique can be used in any MPS and Tree-Tensor Network problem [14], but it's particular utility in open-system problems has only recently been appreciated. We shall make use of this technique in this article, but a discussion of MPS, TDVP and other computational aspects is left to the dedicated presentations in the literature [26-29].

Instead, the key issue that we wish to explore in this study is the remarkable recent result of Tamescelli et al. [30] that allows wave function approachs to OQS to effectively capture the effects of finite temperature environments through the simulation of an equivalent zero-temperature proxy system. As already discussed above, in the non-perturbative, non-markovian regime of OQS, computing the evolution of the single wave function from a sharp initial condition can already be very demanding: converging results over the astronomically large space of initial conditions in a thermal ensemble rapidly becomes imposible. If we also wish to explore the role of non-classical effects in heat flows between finite-temperature environments, the problem becomes exponentially worse. The access to finite temperature properties from a single zero-temperature (pure) wave function simlation thus opens up an entire class of powerful non-perturbative methods for the study of novel open quantum systems. This work aims to establish the extent to which this Tamescelli's 'T-TEDOPA' theory translates into affordable non-perturbative TDVP simulations of thermal and non-equilibrium OQS dynamics, as well as to explore some of the non-classical and non-additive aspects of heat exchange in OQS.

This article is organised as follows. In Section 2.1 we present the Spin-boson Hamiltonians that we will simulate. Sections $2.2 \& 2.3$ give a summary of the T-TEDOPA theory that we will employ in our numerical investigations. Section 2.4 then presents a careful study of the non-perturbative spin-boson model at finite temperatures that reveals some of practical numerical costs implicit in this approach. Thanks to this testing, we are able to offer a freely accessible data set that can be used as a benchmark 
for other numerical approaches to this model, as well as code packages that allow users to perform their own TDVP calculations on finite-temperature open systems. In anticipation of the need to explore non-equilibrium states in a wide range of future contexts, we go on to test the non-perturbative physics of a two-level system (TLS) coupled to two environments at different temperatures in Section 2.5. Exploiting the information in the many body system-environment(s) wave function, we examine the microscopic behaviour of the heat flows between the system and the environments as a function of environmental coupling strength and temperature differences, and highlight a number of non-additive effects arising from non-perturbative quantum polaron effects. Finally, we summarise and discuss our findings in Section 3.

\section{Results}

\subsection{Model, parameters and initial conditions}

We shall shall base our exploration of finite temerperature open dynamics through numerical simulations and analysis of a quantum two-level system that is strongly coupled to either one or two bosonic baths at different temperatures, as illustrated in Figure 1a. The two baths are labeled $a$ and $b$ and are at different inverse temperatures $\beta_{a}$ and $\beta_{b}$, respectively $\left(\beta=1 /\left(k_{b} T\right)\right)$. The system-bath Hamiltonian is given by

$$
\hat{H}=\frac{\omega_{0}}{2} \sigma_{z}+\hat{H}_{I}^{a}+\hat{H}_{I}^{b}+\hat{H}_{B}^{a}+\hat{H}_{B}^{b}
$$

where

$$
\begin{aligned}
\hat{H}_{I}^{a} & =\sigma_{x} \otimes \sum_{k}\left(g_{k}^{*} \hat{a}_{k}+g_{k} \hat{a}_{k}^{\dagger}\right) \\
\hat{H}_{I}^{b} & =\sigma_{x} \otimes \sum_{k}\left(g_{k}^{*} \hat{b}_{k}+g_{k} \hat{b}_{k}^{\dagger}\right) \\
\hat{H}_{B}^{a} & =\sum_{k} \omega_{k} \hat{a}_{k}^{\dagger} \hat{a}_{k} \\
\hat{H}_{B}^{b} & =\sum_{k} \omega_{k} \hat{b}_{k}^{\dagger} \hat{b}_{k} .
\end{aligned}
$$

The TLS is described by the standard Pauli operators $\sigma$, while the bath properties are characterised by their spectral density $J(\omega) \equiv \pi \sum_{k}\left|g_{k}\right|^{2} \delta\left(\omega-\omega_{k}\right)$. This continuous function can be take various forms in specific physical realisations such as electron-phonon interactions, emitter-photon or exciton-vibration coupling in molecular systems. It is well known that the qualitative behaviour of the TLS depends sensitively on the form of $J(\omega)$, especially at low temperatures [? ]. For simplicitly, we assume identical system-bath couplings for both environments and use the common linear frequency dependence that defines an Ohmic environment $\omega_{c}$

$$
J(\omega)=2 \pi \alpha \omega \theta\left(\omega_{c}-\omega\right),
$$

where $\alpha$ is a dimensionless coupling constant and we have introduced a hard frequency cut-off $\omega_{c}$.

The initial condition $\hat{\rho}(0)$ for our numerical simulations is taken to be an uncorrelated (product) state of the spin and baths, which - because of the baths' finite temperatures - must be described by a mixed state, i.e. a density matrix

$$
\hat{\rho}(0)=\rho_{s} \otimes \frac{e^{-\hat{H}_{B}^{a} \beta^{a}}}{\operatorname{Tr}\left\{e^{-\hat{H}_{B}^{a} \beta^{a}}\right\}} \otimes \frac{e^{-\hat{H}_{B}^{b} \beta^{b}}}{\operatorname{Tr}\left\{e^{-\hat{H}_{B}^{b} \beta^{b}}\right\}},
$$

where $\rho_{s}$ is some arbitrary initial density matrix for the TLS. 
Remarkably, despite the initial condition containing two statistically mixed thermal density matrices, it has recently been shown by Tamescelli et al. that the reduced dynamics of the spin can still be obtained from a single simulation of an equivalent pure, i.e. zero temperature, system-environment wave function $[30,31]$. As this result is central for generating our numerical results and our later discussion, we shall now give a brief summary of the protocol first presented in Ref. [30].

\subsection{Finite-temperature reduced dynamics from pure wave function evolution}

In this section we shall closely follow the original notation and presentation of Tamascelli et al [30] and, to simplify the presentation, we shall only consider the coupling to a single environment. The procedure can be easily generalized to multiple environments. Our starting point is the generic Hamiltonian for a system coupled to a bosonic environment consisting of a continuum of harmonic oscillators

$$
H_{S E}=H_{S}+H_{E}+H_{I}
$$

where

$$
H_{I}=A_{S} \otimes \int_{0}^{\infty} d \omega \hat{O}_{\omega}, H_{E}=\int_{0}^{\infty} d \omega \omega a_{\omega}^{\dagger} a_{\omega}
$$

The Hamiltonian $H_{S}$ is the free system Hamiltonian and $A_{S}$ is a generic system operator which couples to the bath. For the bath operators we take the displacements

$$
O_{\omega}=\sqrt{J(\omega)}\left(a_{\omega}+a_{\omega}^{\dagger}\right)
$$

thus defining the spectral density $J(\omega)$. This has been written here as a arbitrary continuous function, but we note that the formulism can also be applyied to in the case of coupling to a discrete set of vibrational modes by adding suitable structure to the spectral density, i.e. sets of lorentzian peaks or Dirac functions [32-34].

The state of the system+environment at time $t$ is described by a mixed state described by a density matrix $\rho_{S E}(t)$. The initial condition is assumed to be a product of system and environment states $\rho_{S E}(0)=\rho_{S}(0) \otimes \rho_{E}(0)$ where $\rho_{S}(0)$ is an arbitrary density matrix for the system and $\rho_{E}(0)=\exp \left(-H_{E} \beta\right) / \mathcal{Z}$, with the environment partition function given by $\mathcal{Z}=\operatorname{Tr}\left\{\exp \left(-H_{E} \beta\right)\right\}$. Such a product state is commonly realised in non-equilibrium problems where the environment equilibrium configuration corresponds to the sub-system's unexcited state, as is routinely encountered in photophysics or quantum quenches $[35,36]$. The environment thus begins in a thermal equilibrium state with inverse temperature $\beta$, and the energy levels of each harmonic mode are statistically populated. For a very large (continuum) of modes, the number of possible thermal configurations grows extremely rapidly with temperature, essentially making it impossible to obtain a converged sampling of these configurations when each instance involves demanding wave function simulations. We briefly note that some more effficient sampling methods involving sparse grids and/or stochastic mean-field approaches have recently been proposed and demonstrated [37,38], as well as some effective MPS techniques for capturing finite temperatue effects in frequency domain simulations [39].

The initial thermal condition of the environmental oscillators is also a Gaussian state, for which is it further known that the influence functional [1] - which is a full description of the influence of the bath on the system - will depend only on the two-time correlation function of the bath operators

$$
S(t)=\int_{0}^{\infty} d \omega\left\langle O_{\omega}(t) O_{\omega}(0)\right\rangle
$$

Any two environments with the same $S(t)$ will have the same influence functional and thus give rise to the same reduced system dynamics, i.e. the same $\rho_{S}(t)=\operatorname{Tr}\left\{\rho_{S E}(t)\right\}$. That the reduced systems dynamics are completed specificied by the spectral density and temperature of a gaussian environment has been know for a long time [1], but the key idea of the equivalance - and thus the possibly of the 
interchange - of environments with the same correlation functions has only recently been demonstrated by Tamascelli et al. [40].

The time dependence in eq. 11 refers to the interaction picture so that the bath operators evolve under the free bath Hamiltonian: $O_{\omega}(t)=e^{i H_{E} t} O_{\omega}(0) e^{-i H_{E} t}$. Using eq. 10 and $\left\langle a_{\omega}^{\dagger} a_{\omega}\right\rangle=n_{\beta}(\omega)$ we have

$$
S(t)=\int_{0}^{\infty} J(\omega)\left[e^{-i \omega t}\left(1+n_{\beta}(\omega)\right)+e^{i \omega t} n_{\beta}(\omega)\right] .
$$

Making use of the relation

$$
\frac{1}{2}(1+\operatorname{coth}(\omega \beta / 2)) \equiv\left\{\begin{array}{l}
n_{\omega}(\beta), \omega \geq 0 \\
-\left(n_{|\omega|}(\beta)+1\right), \omega<0
\end{array}\right.
$$

we can write eq. 12 as an integral over all positive and negative $\omega$

$$
S(t)=\int_{-\infty}^{\infty} d \omega \operatorname{Sign}(\omega) \frac{J(|\omega|)}{2}\left(1+\operatorname{coth}\left(\frac{\omega \beta}{2}\right)\right) e^{-i \omega t} .
$$

But eq. 14 is exactly the two-time correlation function one would get if the system was coupled to a bath, now containing positive and negative frequencies, at zero temperature! The effects of the finite, physical temperature now appear in a new effective spectral density for the extended environment given by

$$
J_{\beta}(\omega)=\operatorname{Sign}(\omega) \frac{J(|\omega|)}{2}\left(1+\operatorname{coth}\left(\frac{\omega \beta}{2}\right)\right)
$$

Thus, we find that our open system problem is completely equivalent to the one governed by the Hamiltonian

$$
H=H_{S}+H_{E}^{\mathrm{ext}}+H_{I}^{\mathrm{ext}},
$$

in which the system couples to an extended environment, where

$$
\begin{aligned}
& H_{I}^{\mathrm{ext}}=A_{S} \otimes \int_{-\infty}^{\infty} d \omega \sqrt{J_{\beta}(\omega)}\left(a_{\omega}+a_{\omega}^{\dagger}\right), \\
& H_{E}^{\mathrm{ext}}=\int_{-\infty}^{\infty} d \omega \omega a_{\omega}^{\dagger} a_{\omega},
\end{aligned}
$$

and which has the initial condition $\rho_{S E}(0)=\rho_{S}(0) \otimes|0\rangle_{E}\langle 0|$. This transformed initial condition is now far more amenable to simulation as the environment is now described by a pure, single-configuration wave function, rather than a statistical mixed state, and so no statistical sampling is required to capture the effects of temperature on the reduced dynamics!

Analysing the effective spectral density of Eq. 15, it can be seen that the new extended environment has thermal detailed balance between absorption and emission processes encoded in the ratio of the coupling strengths to the positive and negative modes in the extended Hamiltonian (see Figure1c), as opposed to the operator statistics of a thermally occupied state of the original, physical mode, i.e.

$$
\frac{J_{\beta}(\omega)}{J_{\beta}(-\omega)}=\frac{\left\langle a_{\omega} a_{\omega}^{\dagger}\right\rangle_{\beta}}{\left\langle a_{\omega}^{\dagger} a_{\omega}\right\rangle_{\beta}}=e^{\beta \omega}
$$

Indeed, from the system's point of view, there is no difference between the absoprtion of a quantum from a thermally occupied, positive-energy bath mode and the creation (emission) of an excitation into an unoccupied, negative energy, bath mode. The extension to negative frequencies essenitally allows the process whereby the system would be heated by the environment (absorpting pre-existing quanta in the thermal bath) to be mimicked by spotaneous emission into a negative energy vacuum of states, as shown in Figure 1b. 
In fact, the equivalence between these two environments goes beyond the reduced system dynamics as there exists a unitary transformation which links the extended environment to the original thermal environment. This means that one is able to reverse the transformation and calculate thermal expectations for the actual bosonic bath such as $\left\langle a_{\omega}^{\dagger}(t) a_{\omega}(t)\right\rangle_{\beta}$. This is particularly useful for molecular systems in which environmental (vibrational) dynamics are also important observables that report on the mechanisms and pathways of physio-chemical transformations [41-43]. In this article, we will use this capability later to look at the non-equilibrium heat flows between the TLS and it's environments. This is a major advantage of many body wavefunction approaches, as full information about the environment is available, c.f. effective master equation descriptions which are obtained after averaging over the environmental state.

(a)

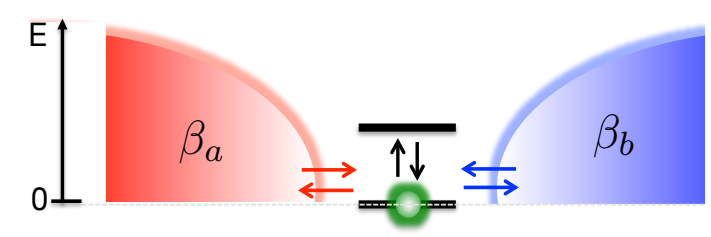

(b)

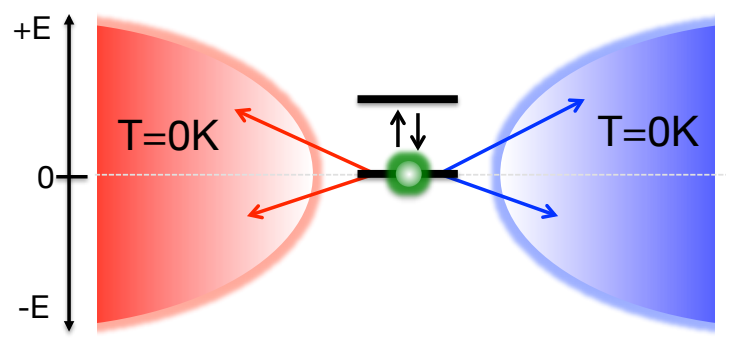

(c)

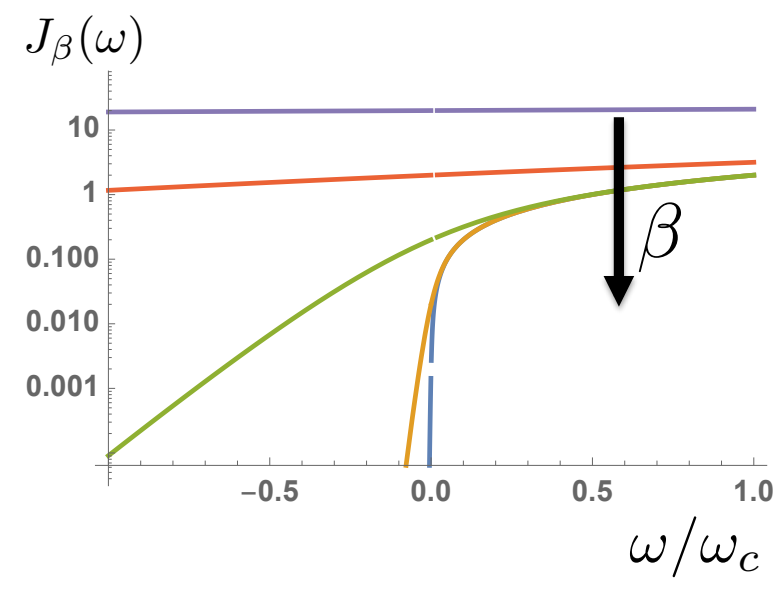

Figure 1. (a) Two-level system (TLS) is coupled to two environments $(a, b)$ with inverse temperatures $\beta_{a}$ and $\beta_{b}$. (b) The reduced state dynamics of the TLS can be obtained from a zero-temperature simulation of an extended environment containing negative frequency excitation modes and temperature-dependent couplings. (c) The effective spectral density $J_{\beta}(\omega)$ encodes the principle of detailed balance for absrption and emission of quanta between thermal transitions in the TLS. For the Ohmic spectral density considered in this article, $J_{\beta}(\omega)$ becomes flat over the entire range $\left[-\omega_{c}, \omega_{c}\right]$ as the temperature increases ( $\beta$ decreases). 


\subsection{Chain mapping and chain coefficients}

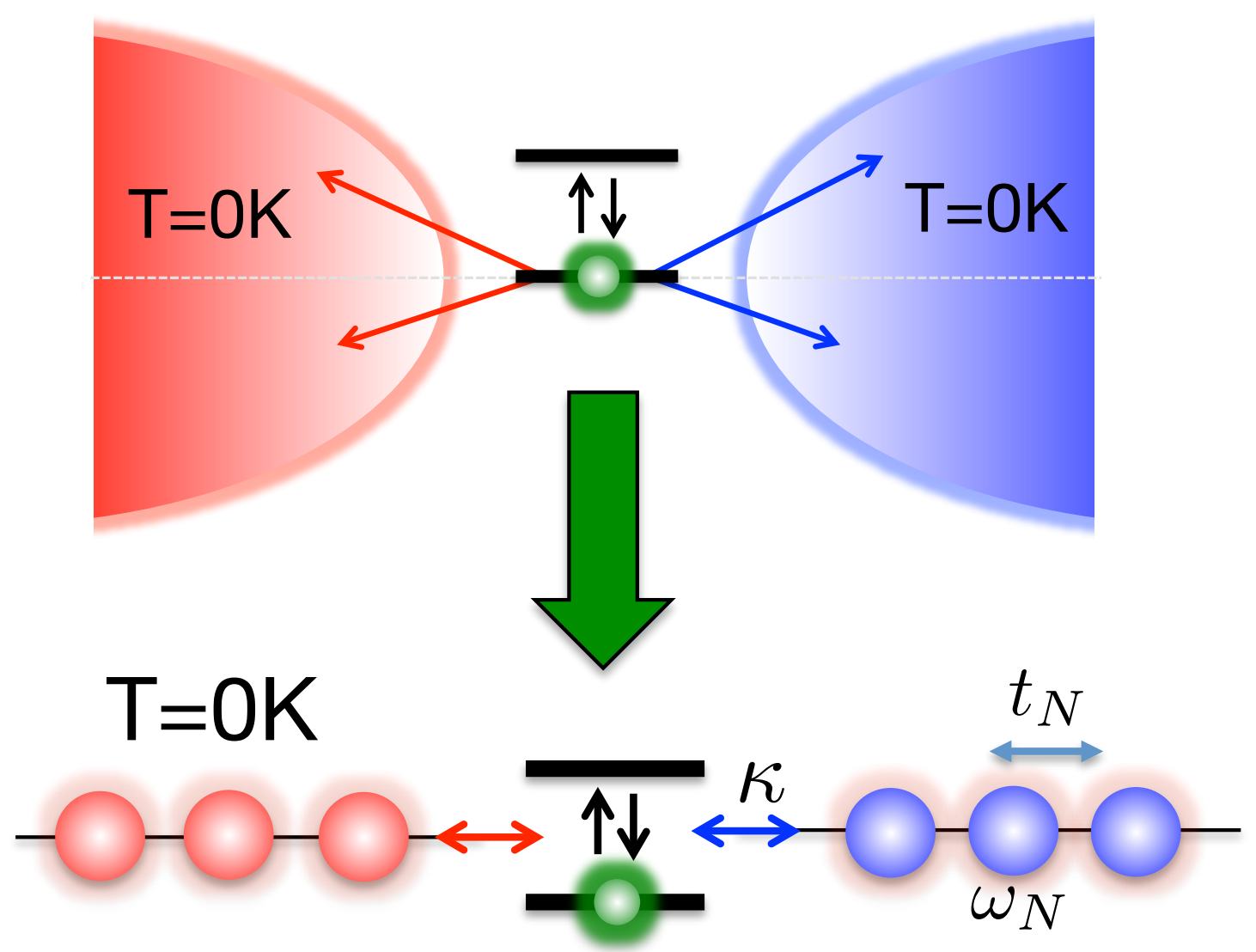

Figure 2. o

Following this transformation a further step is required to facilitate efficient simulation of the many-body system+environment wave-function. This is to apply a unitary transformation to the bath modes which converts the star-like geometry of $H_{I}^{\text {ext }}$ into a chain-like geometry, thus allowing the use of Matrix-Product-State (MPS) methods $[10,44,45]$ (see Figure2). We thus define new modes $c_{n}^{(+)}=\int_{-\infty}^{\infty} U_{n}(\omega) a_{\omega}^{(+)}$, known as chain modes, via the unitary transformation $U_{n}(\omega)=\sqrt{J_{\beta}(\omega)} p_{n}(\omega)$ where $p_{n}(\omega)$ are orthonormal polynomials with respect to the measure $d \omega J_{\beta}(\omega)$. Thanks to the three term recurrence relations associated with all orthonormal polynomials $p_{n}(\omega)$, only one of these new modes, $n=1$, will be coupled to the system, while all other chain modes will be coupled only to their nearest neighbors [44]. Our interaction and bath Hamiltonians thus become

$$
\begin{aligned}
& H_{I}^{\text {chain }}=\kappa A_{S}\left(c_{1}+c_{1}^{\dagger}\right), \\
& H_{E}^{\text {chain }}=\sum_{n=1}^{\infty} \omega_{n} c_{n}^{\dagger} c_{n}+\sum_{n=1}^{\infty}\left(t_{n} c_{n}^{\dagger} c_{n+1}+\text { h.c }\right) .
\end{aligned}
$$

The chain coefficients appearing in eq. 19 are related to the three-term recurrence parameters of the orthonormal polynomials and can be computed using standard numerical techniques [44]. Since the initial state of the bath was the vacuum state, it is unaffected by the chain transformation. We briefly note the evolution of the asymptotic values of the chain parameters, as illustrated in Figure ??. For a smooth spectral density with a hard cut-off, it is has been rigorously proven that $\omega_{n} \rightarrow$ $\omega_{c} / 2, t_{n} \rightarrow \omega_{c} / 4$ as $n \rightarrow \infty$ [? ]. Figure ?? shows the dramatic changes in these asymptotic values as the temperature is increased, which - from the numerical results show in Figure ?? appear to be 
$\omega_{n} \rightarrow 0, t_{n} \rightarrow \omega_{c} / 2$ as $n \rightarrow \infty$ and $\beta \rightarrow 0$. This can be naturally understood from the behaviour of the effective spectral functions $J_{\beta}(\omega)$ with increasing temperature, as illustrated in Figure1c. The spectral functions become symmetric and have finite values over the whole domain $\left[-\omega_{c}, \omega_{c}\right]$. The asymptotic spectrum of the chain modes thus has a bandwidth of $2 \omega_{c}$ centered on $\omega=0$, which, for a uniform hopping chain, requires the numerically observed asymptotic chain parameters. In the particular case of the Ohmic environment at high temperatures, is can easily be seen that $J_{\beta}(\omega)$ tends to a constant and so will have a chain representation derived from the classical Legendre polynomials [46].

We have thus arrived at a formulation of the problem of finite-temerature open systems in which the many-body environmental state is initialised as a pure product of trivial ground states, whilst the effects of thermal flucutations and populations are encoded in the Hamiltonian chain parameters and system-chain coupling. These parameters must be determined once for each temperature but - in principle - the actual simulation of the many body dynamics is now no more complex than a zero-temperature simulation! 

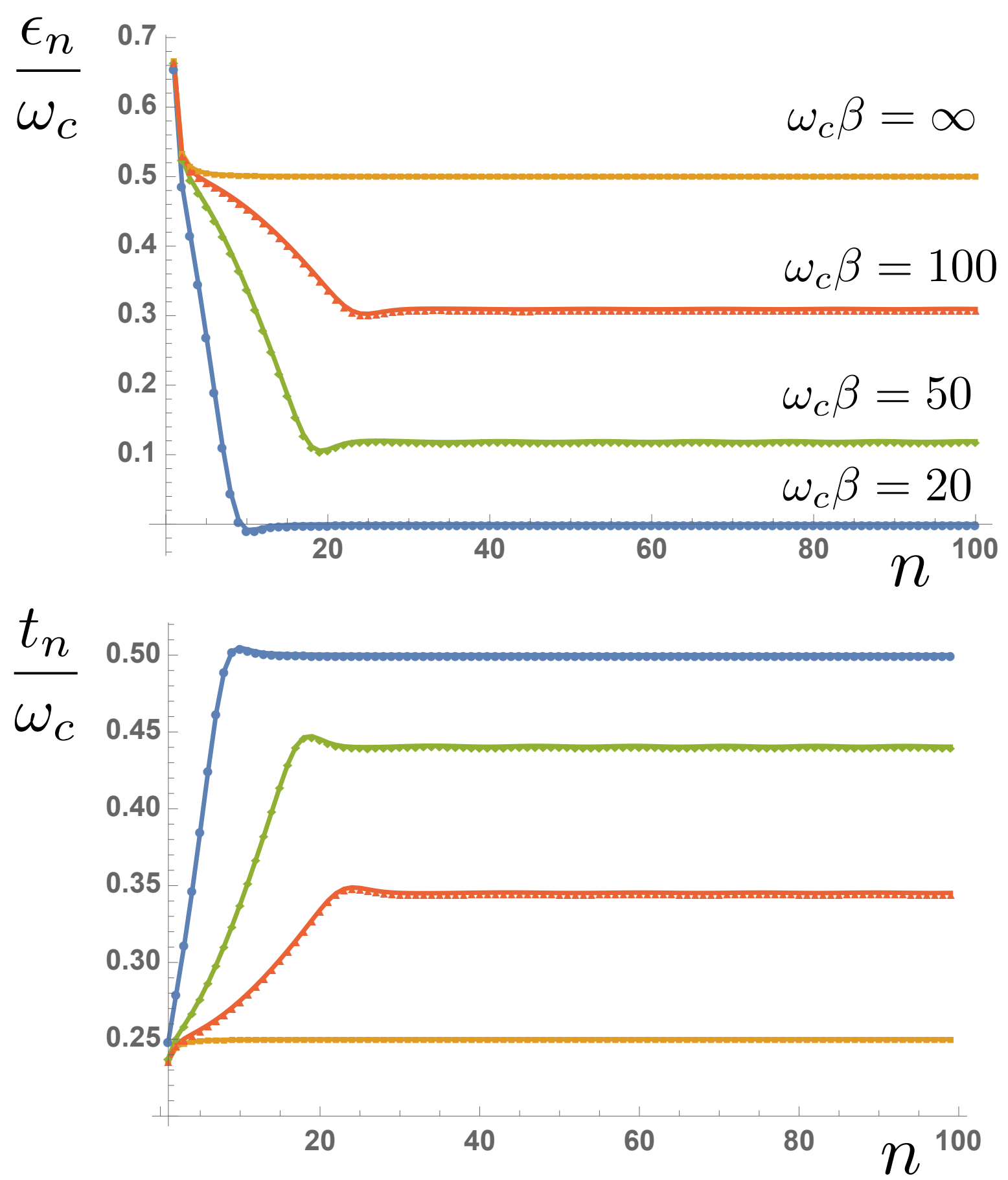

Figure 3. Site energies $e_{n}$ (a) and hopping amplitudes $t_{n}(\mathrm{~b})$ as a function of chain distance $n$ at different environment temperatures

\subsection{Spin-boson model across the complete $\alpha-\beta$ space}

In this section, we numerically verify that the finite-temperature approach set out in Sections ?? \& 2.3 captures the correct non-perturbative behaviour in the single-bath spin-boson model. This will be illustrated with a few explicit examples, but the key result of this section is the creation of a comprehensive dataset for the Ohmic spin-boson model that allows arbitrary TLS initial conditions to be propagated in real-time and over a large area of $\alpha-\beta$ space. This data set, which we have made freely available in the SI of the article, can be used to benchmark other methods and for applications.

Figure $4 \mathrm{a}$, b shows the temporal decay of an initially polarized spin $\left\langle\sigma_{z}(0)\right\rangle=+1$ towards thermal equilibirum for varying coupling strengths $\alpha$ and inverse temperatures $\beta$, respectively. The TLS energy 
splitting was $\omega_{0}=0.2 \omega_{c}$. The key result in Figure $4 \mathrm{~b}$ is the dependence of the thermalized spin polarization at long times. In a simple, perturbative rate equation treatment, this final polarization would be set by the energy gap $\omega_{0}$ and the temperature, according to the Gibbs-Boltzmann distribution

$$
\left\langle\sigma_{z}\right\rangle_{\beta}=-\frac{\left(1-e^{-\beta \omega_{0}}\right)}{\left(1+e^{-\beta \omega_{0}}\right)} .
$$

The coupling strength $\alpha$ would only alter the rate at which this thermal distributiuon is reached. However, Figure 4a shows a growing dependence of the final polarization on the coupling strength, suggesting a non-perturbative effect. This is indeed the case: strong coupling leads to polaron formation and non-perturbative renormalisation of the TLS energy gap $\omega_{0}$. According to the variational theory of Silbey and Harris [47], the renormalized gap $\omega_{r}$ is approximatively given by

$$
\omega_{r}=\omega_{0}\left(\frac{\omega_{0}}{\omega_{c}}\right)^{\frac{\alpha}{1-\alpha}}
$$

in the so-called scaling limit in which $\omega_{c}$ is much larger than all other energy scales in the problem. This renormaization is highly non-perturbative, and can completely close the TLS energy gap at a critical coupling $\alpha_{c}=1$ [1].

Accounting for these renormalization effects, an approximate expression for $\left\langle\sigma_{z}\right\rangle_{\beta}$ is

$$
\left\langle\sigma_{z}\right\rangle_{\beta}=-\left(\frac{\omega_{0}}{\omega_{c}}\right)^{\frac{\alpha}{1-\alpha}} \frac{\left(1-e^{-\beta \omega_{r}}\right)}{\left(1+e^{-\beta \omega_{r}}\right)} .
$$

Figure 5 shows this analytical prediction as a function of temperature, compared to the results extracted from the real-time dynamics. As mentioned above, most analytical predictions for the SBM are obtained deep in the scaling limit, while numerical results necessarily involve only moderately large values of $\omega_{c}$. When comparing results, it is common in the literature to evaluate analytical expressions with a rescaled coupling stength $\tilde{\alpha}=c \alpha$ to account for this, which we have applied in Figure 5. We found that a constant factor $c=0.66$ gave excellent agreement across the parameter space for both one and two-bath SBMs, as shown in the inset of Figure 4. 

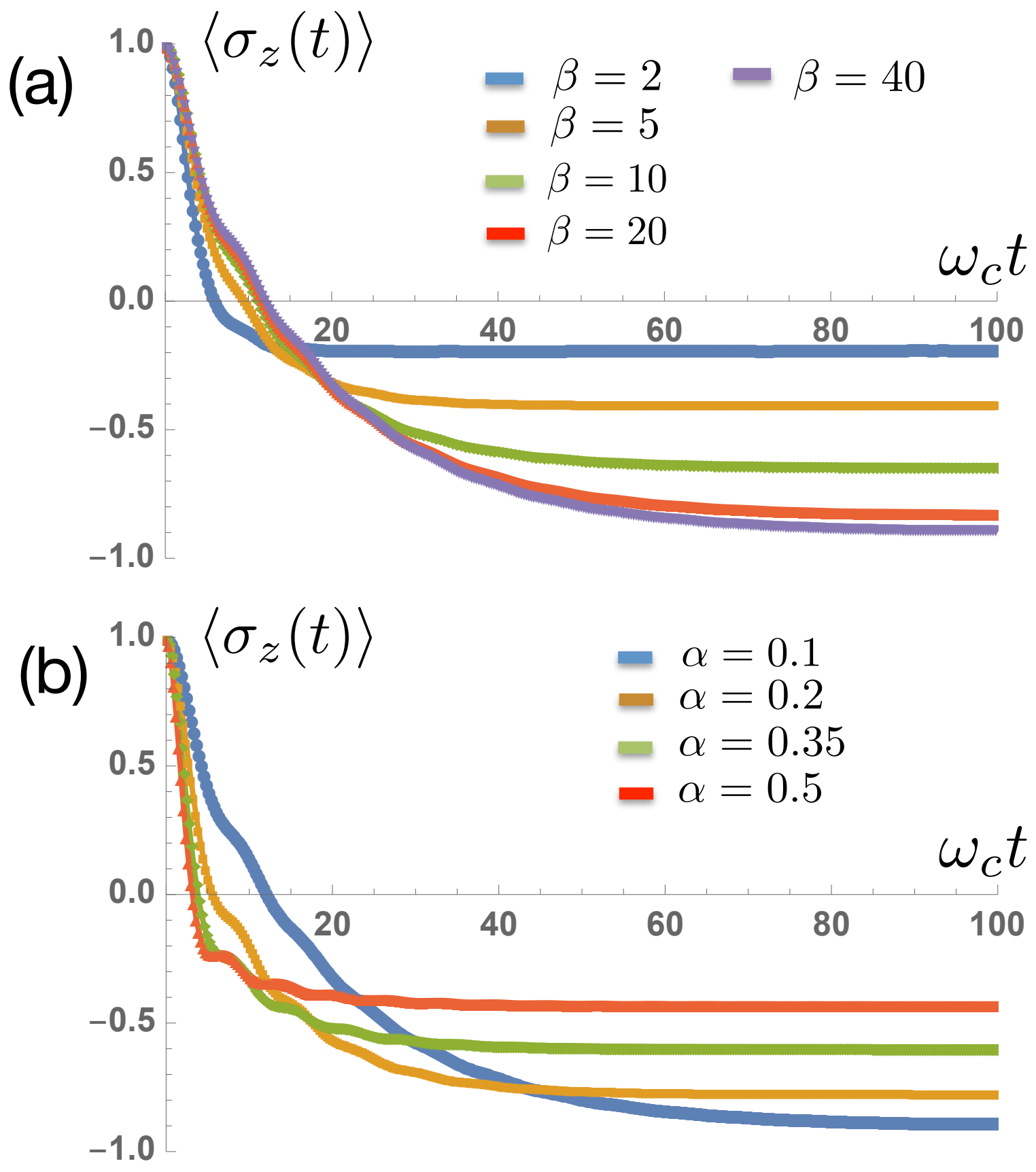

Figure 4. Relaxation of spin polarization as a function of time for (a) different temperatures and $\alpha=0.1$ and (b) different coupling strengths with a fixed $\omega_{\mathcal{c}} \beta=100$. 


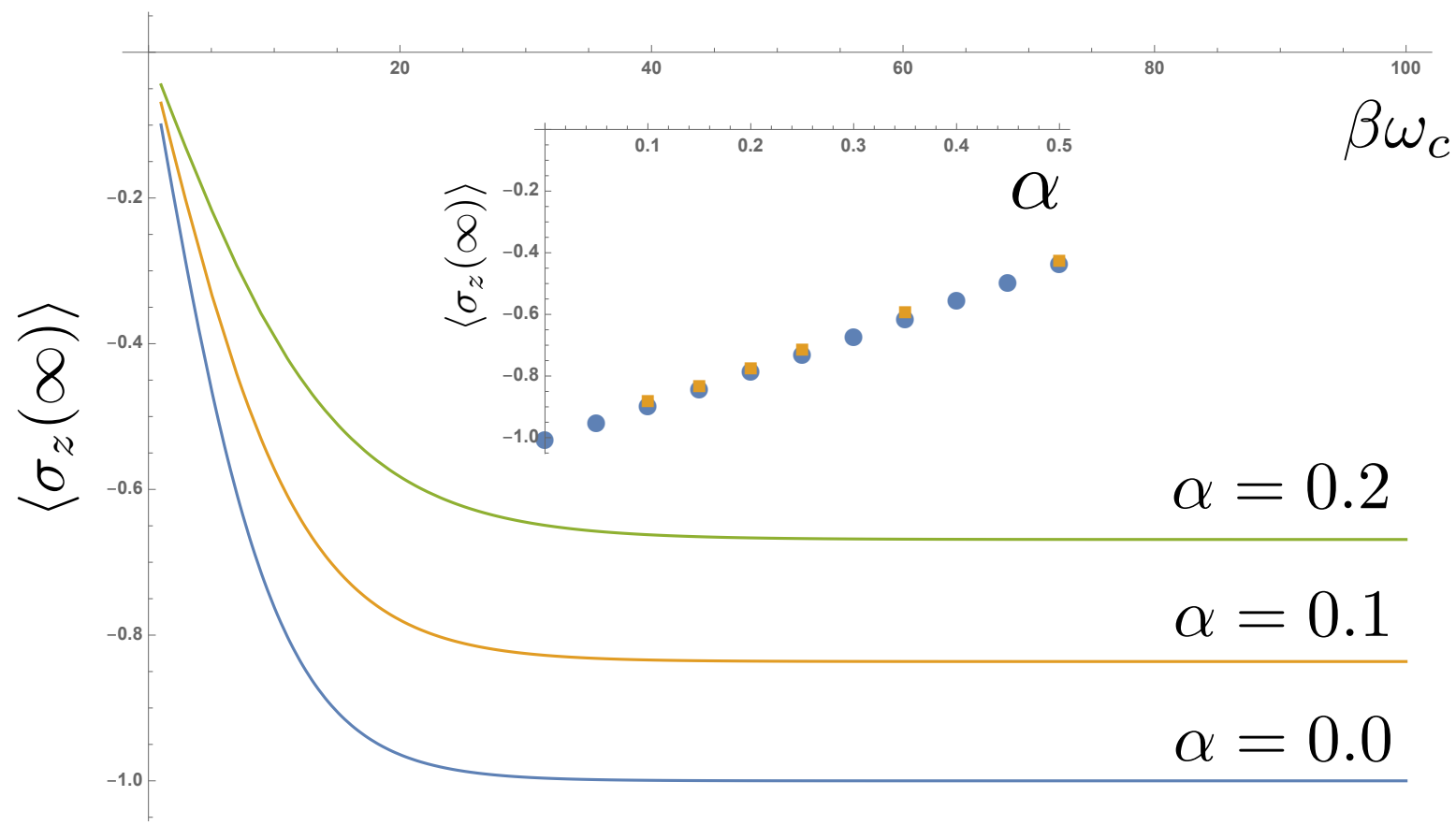

Figure 5. Analytical prediction of thermal steady state spin polarization as a function of inverse temperature $\omega_{c} \beta$. Inset compares these predictions with steady values extracted from the real-time dynamics shown in Figure 4.

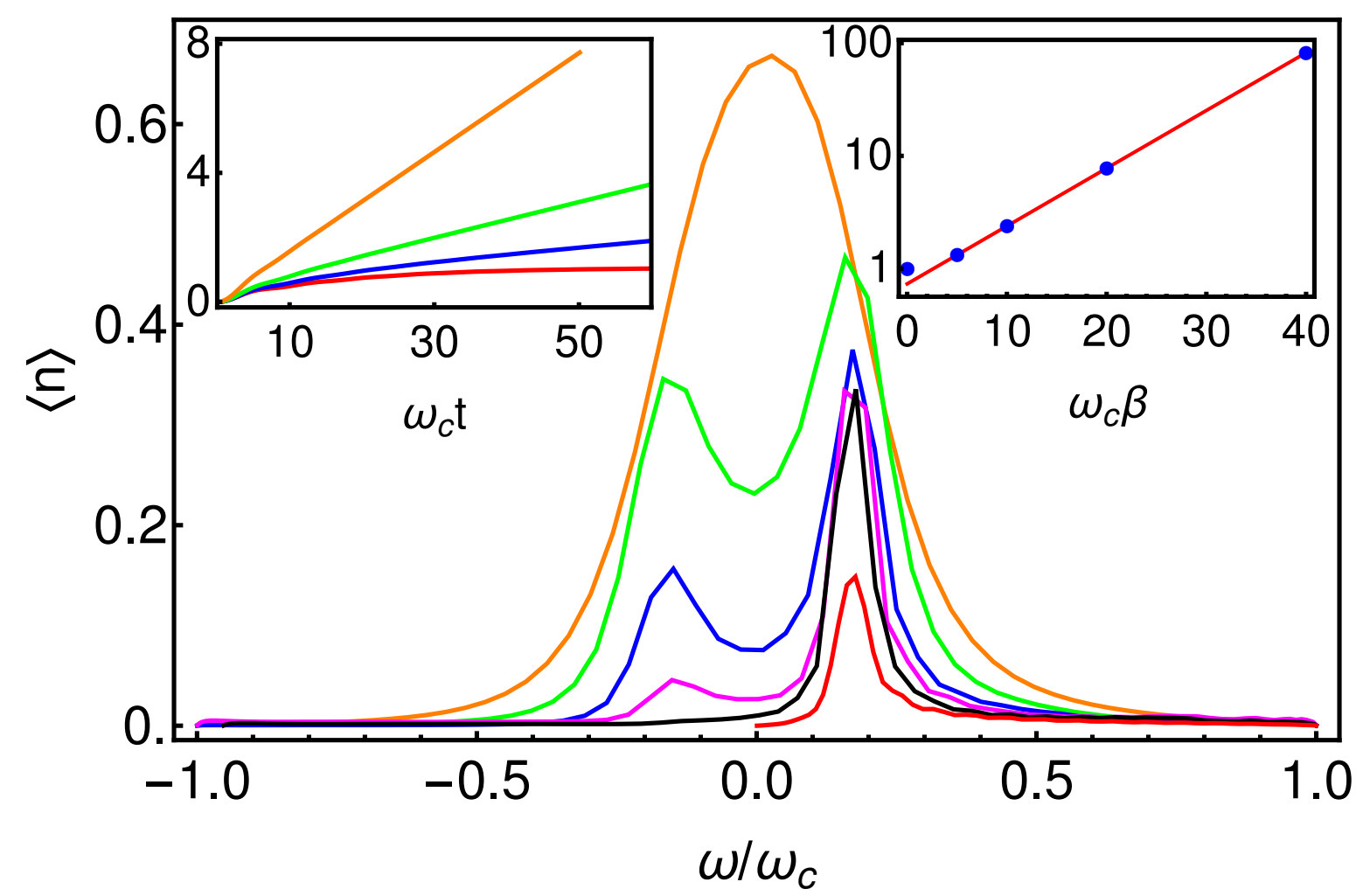

Figure 6. Long-time occupations of the modes of the extended environment, following the thermailization of the TLS at various temperatures. Inset shows the total number of quanta in the environment as a function of time (left) for diffferent temperatures. This population grows indefinitely at finite temperatures. The inset (right) shows the long-time ratio of the peak heights in each curve of the main figure. These give a very good fit to the exponential dependence expected for absoprtion and emission rates obeying detailed balance. 
As a final set of observations in this section, we now look at the behaviour of the environment. In Figure 6 we present the occupations of the bath modes in the extended spectral representation used to account for finite temperatures in Figure 6. As anticipated in our discussion in Section 2.2, we find that at low temperatures, the energy released from the decay of the spin is absoprbed by modes with positive frequencies matching the TLS energy gap $\omega_{0}$. As the temperature increases, peaks appear at negative frequencies, corresponding to the excitation of these modes due to 'heating' of the TLS, i.e. the TLS is thermally excited and removes energy from the environment. As a function of temperature, the ratio of the positive and negative ocupations is a very close fit to $e^{\beta \omega_{0}}$, as expected from detailed balance. However, due to the presence of negative frequency modes, we find that the populations in both the positive and negative frequency modes grow indefinitely during the simulation time, as shown in the inset of Figure 6. The difference of these growing populations plateaus at a finite value, corresponding to the thermal occupation of the physical positive-frequency mode, but care must be taken to get converged results for long-time (steady state) quantities due to the expanding local Hilbert spaces needed for the environment modes in the simulations.

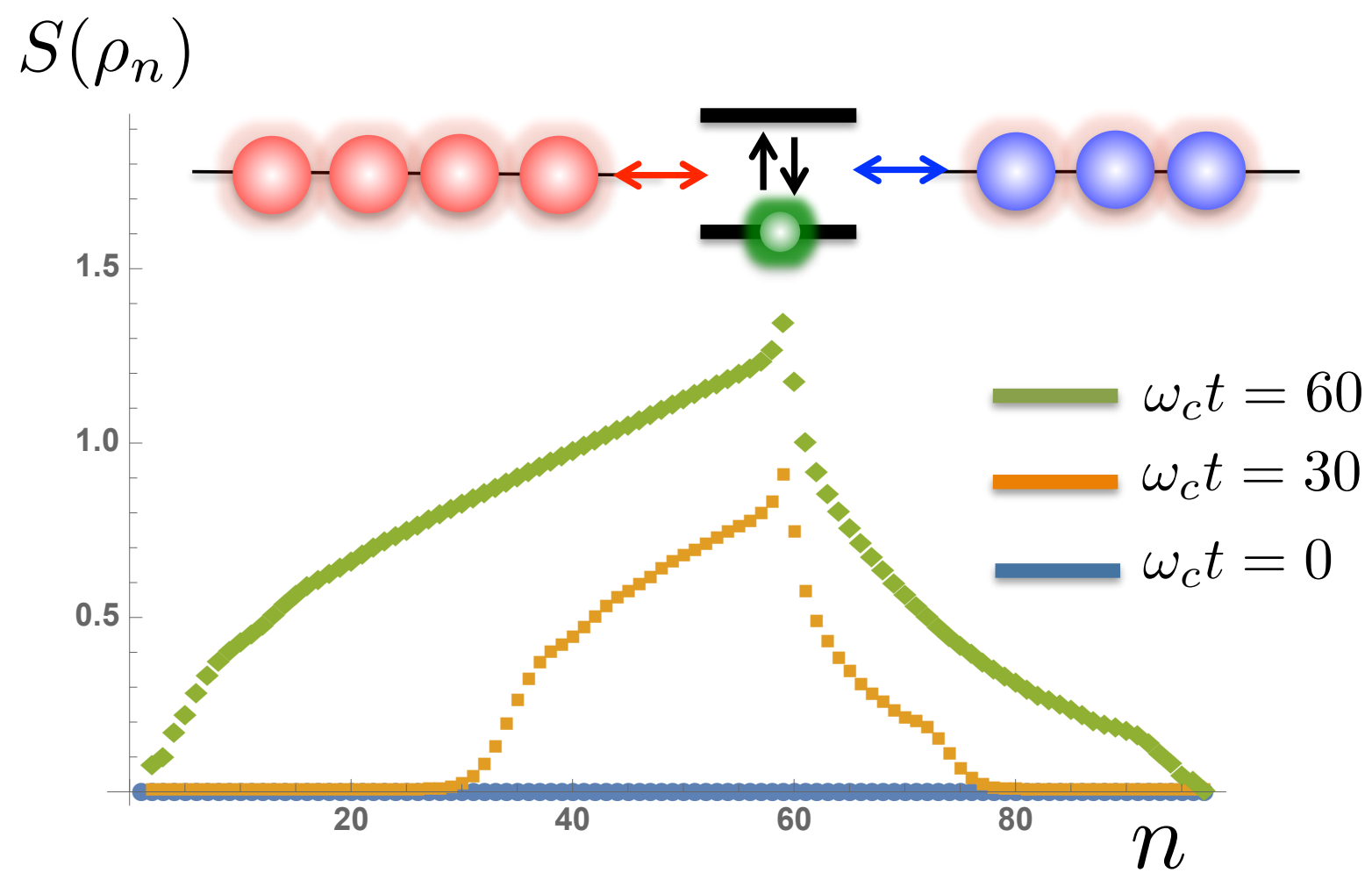

Figure 7. The time evolution of the Von Neumann entanglement entropy for each bipartition of the system-environment chain at site $n$. The TLS is located in this example at site $n=59$, with the hot bath correspnding to sites $1-58$ and remaining sites represent the cold bath.

Figure 7 shows the behaviour of the von-Neumann entropy obtained by bi-partitioning the total $N$-site system-environment chain into chains of size $n$ and $N-n$ and computing the singular values of either of the subsystems' reduced density matrices [? ]. The entanglement entropy directly reports on the size of the bond-dimensions required to represent the state accurately in the MPS format, and our results show that this entropy also grows continuously during the simulation. There is also a clear asymmetry in the rate of spreading and magnitudes of entanglement, with correlations between sites in the hot environment growing much faster. Again, these growing numerical resources for finite temperature simulations should be handled with care, and we shall take this up again in Section 3. 


\subsection{Non-equilibrium heat flows}

In this section we simulate the non-equilibrium dynamics of the TLS connected to two environments at different temperatures. For clarity we will designate environment $a$ as the 'hot' environment and $b$ as the 'cold ' one, using suffixes ' $c$ ' and ' $h$ ', respectively. We note here that this elementary class of two-environment models has both wide-ranging practical applications - such as studying heat and charge transfer in nanodevices and molecules [6,48,49], as well as being of fundamental relevance for quantum thermodynamics, decoherence, and non-equilibrium steady states [50-54]. Figure 8a shows the real-time excitation of a TLS initially prepared in its ground state when connected at $t=0$ to the cold environment with fixed $\omega_{0} \beta_{c}=100$ and the 'hot' environment at different temperatures. Figure $8 \mathrm{~b}$ shows the steady-state spin polarization as a function of the temperature difference between the hot and cold baths. To understand the basic features of the steady state, let's consider a perturbative set of rate equations for the population of the spin-up level $P_{\uparrow}(t)$. Assuming that the rates of absorption and emission from each bath of TLS obey detailed balance, the dynamics of $P_{\uparrow}(t)$ can be obtained from the equation

$$
\frac{d P_{\uparrow}(t)}{d t}=-\Gamma P_{\uparrow}(t)\left(n_{c}+n_{h}+2\right)+\Gamma\left(1-P_{\uparrow}(t)\right)\left(n_{c}+n_{h}\right),
$$

where $n_{i}=\left[\exp \left(\omega_{0} \beta_{i}\right)-1\right]^{-1}$ By finding the steady state population $P_{\uparrow}(\infty)$, the non-equilibrium value of the spin polarisation $\left\langle\sigma_{z}^{a b}(\infty)\right\rangle$ then takes the simple form

$$
\left\langle\sigma_{z}^{a b}(\infty)\right\rangle=\frac{n_{h}+n_{c}}{2\left(n_{c}+n_{h}+1\right)} .
$$

. Once again, if renormalization effects are included, the agreement between the analytical predictions is very good, as can be seen in Figure 8b. Indeed, for the lowest temperatures, the spin dyanmics are entirely due to renormalization effects, as thermal occupation of the excited level is negligible.

Interestingly, these two-bath results also reveal an intriguing non-additive effect due to the coupling to two environments. The subject of non-additivity of environmental interactions has recently attracted attention due to the role of multiple environments in a wide range of 'active' quantum machines, such as the conversion of ambient solar energy in room-temperature (phonon-coupled) devices $[15,16]$, and also the highly co-operative actions of different types of vibrational motion in molecular photophysics [14]. In the present case, the non-additive effects appear in the polaronic renormalisation, which is mostly clearly seen in the case when the two baths have the same temperature. This situation is indistinguishable from a coupling to a single bath with twice the coupling strength. The renormalisation can thus be obtained from Eq. 21 with the replacement $\alpha \rightarrow 2 \alpha$. However, the renormalisation arises from the overlap of the displaced mode wave functions that are 'fast ' enough to co-tunnel with the TLS as it transitions between $\left\langle\sigma_{x}\right\rangle= \pm 1$ [47,55], and in an additive approximation the renormalization would be simply be the product of the individual overlaps for each environment. However, as is clear form Eq. 21, the this doubling of the coupling does not lead to a simple exponential doubling of the renormalization, but instead leads to a nonlinear suppression of the energy gap according to the exponent $2 \alpha /(1-2 \alpha)$. 

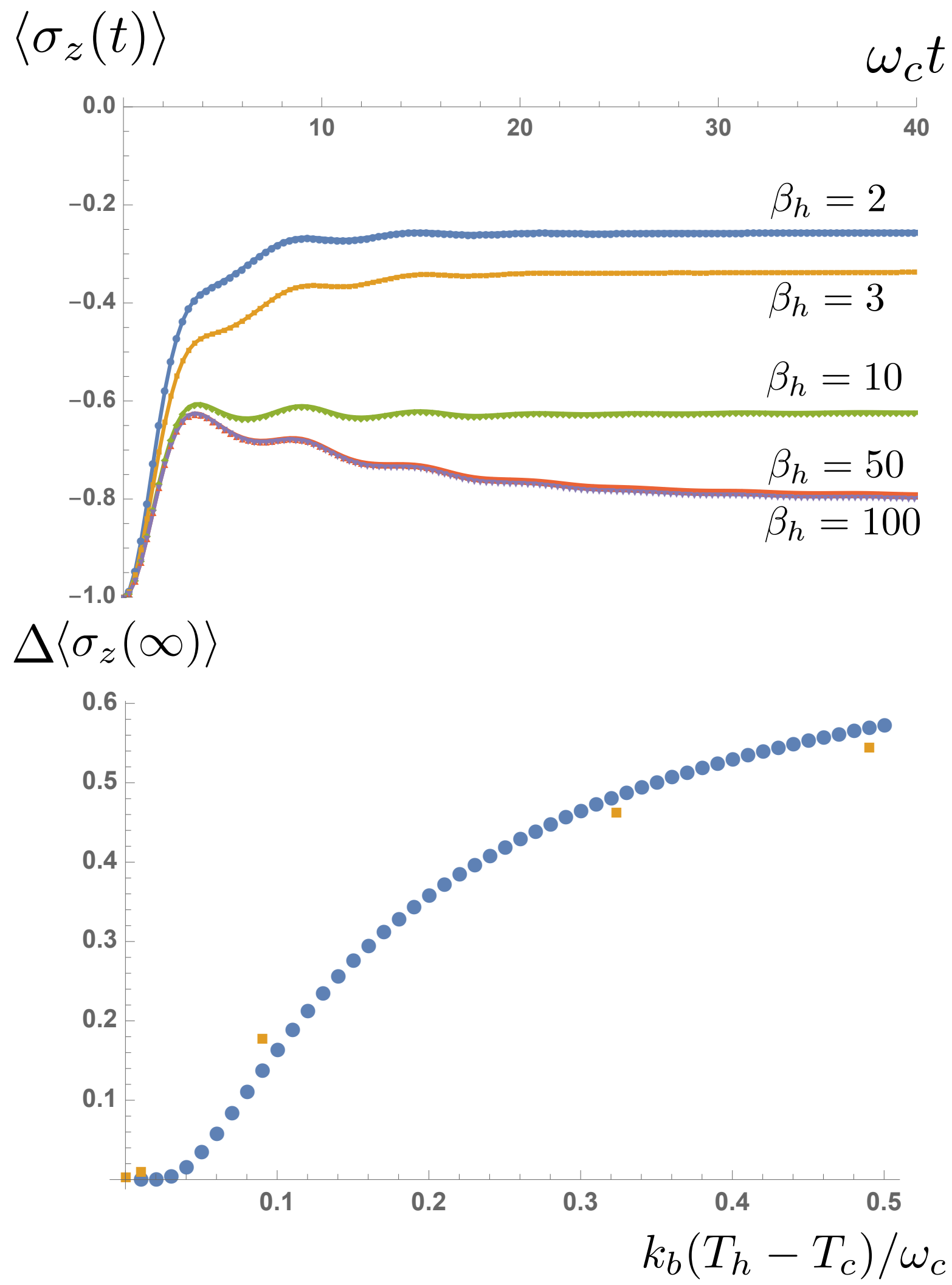

Figure 8. Non-equilibrium relaxation of spin polarization as a function of time for (a) fixed cold bath temperature and varying hot bath temperatures and (b) the change in $\left\langle\sigma_{z}(\infty)\right\rangle$ as a function of the temperature difference bewtween the baths $\left(T_{\mathcal{C}}\right.$ is kept constant). Analytical predictions are shown as dots, numerical data points as squares. 


\section{$\left\langle\sigma_{z}(\infty)\right\rangle$}

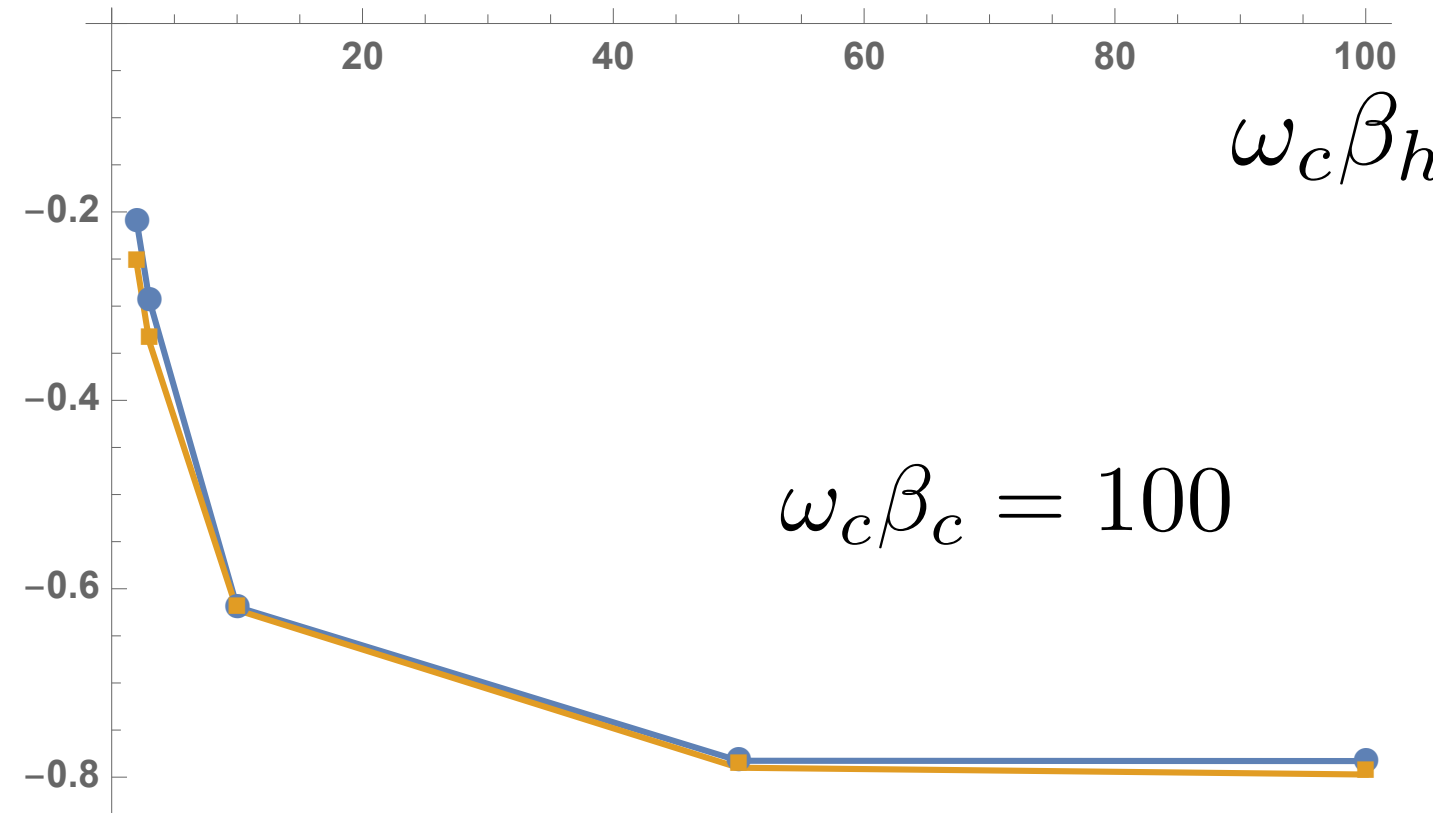

Figure 9. Comparison of non-equilibirum steady state spin polarization obtained from numerical results and analytical predictions.

Exploiting the access to the environmental state, we now show the transient dynamics of the heat flow in the two baths during the establishment of the TLS steady state. We define the following operators

$$
\hat{J}_{c}=\hat{\sigma}_{y} \otimes\left(\hat{A}_{0}^{+}+\hat{A}_{0}\right)
$$

and

$$
\hat{J}_{h}=\hat{\sigma}_{y} \otimes\left(\hat{B}_{0}^{+}+\hat{B}_{0}\right),
$$

which measure the heat flux from the spin to baths $a$ and $b$ respectively. Representative heatflows are shown in Figure ?? for large and zero differences in the bath temperatures. In both cases, the initial dynamics involve heating from both hot and cold environments, as the spin is initially in a pure $(T=0 K)$ groundstate. As the dynamical steady state of the spin is obtained, a net heat current appears from the hot to cold environment. This heat current vanishes as the temperature difference of the baths is reduced, as we would expect. Again, a simple Pauli master equation approach would yield the following prediction for the steady-state heat flux from the hot to cold environment

$$
J=\frac{\Gamma}{2} \frac{n_{h}-n_{c}}{1+n_{h}+n_{c}}
$$

which is plotted aside our numerical data in Figure 11. The simulations correctly capture the essentially non-linear behaviour of heat flow through the quantum 'heat leak' TLS, although a linear regime where Fourier's law of heat flow appears to hold can be clearly observed before the flows saturate for large temperature differences. 


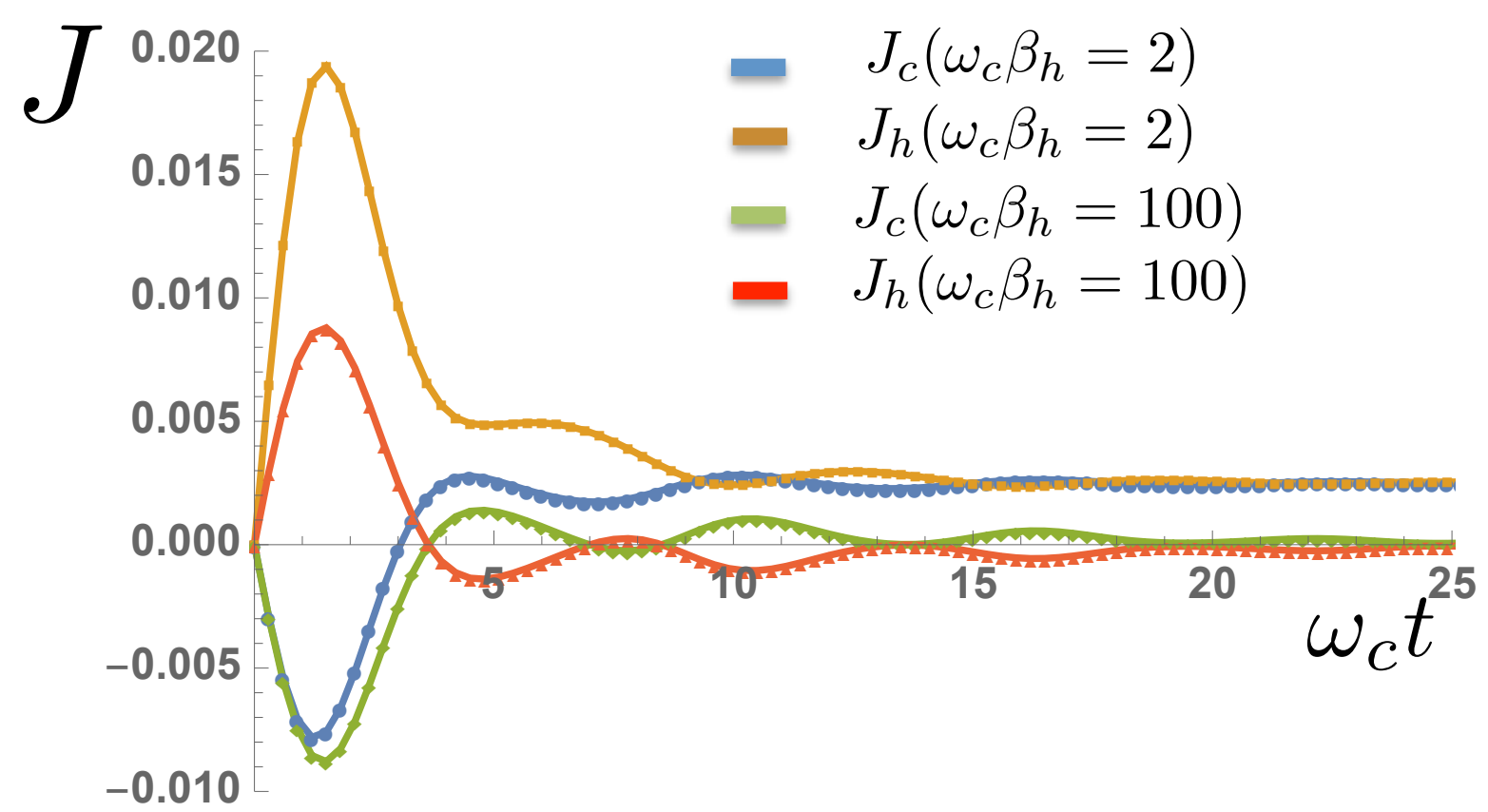

Figure 10. Heat flows into the cold bath $\left(J_{c}\right)$ and out of the hot bath $\left(J_{h}\right)$ as a function of time for varying hot bath temperatues and a fixed $\omega_{c} \beta_{c}=100$.

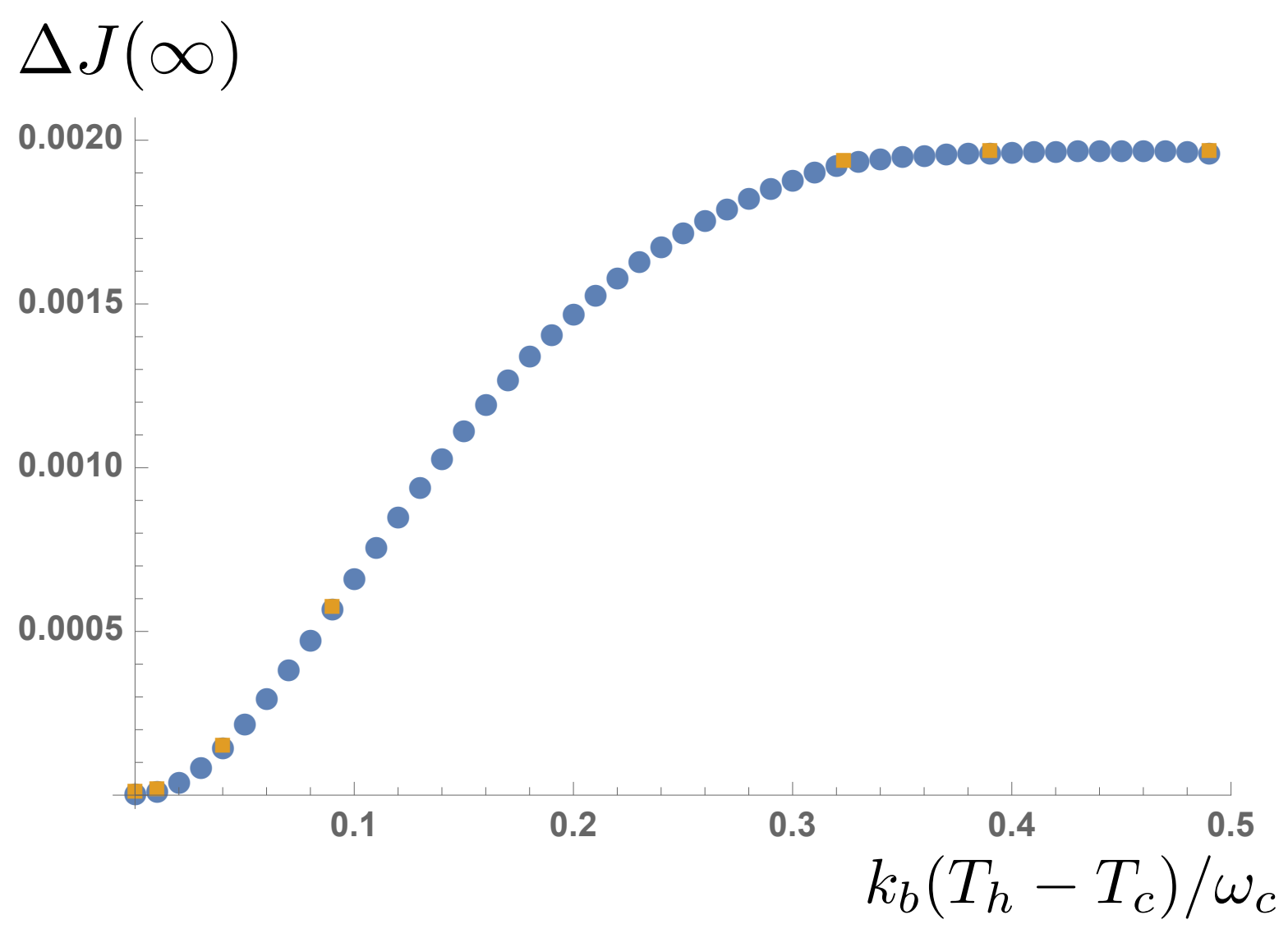

Figure 11. Net steady-state heat flux through the two-level system as a function of temperature diffference for a fixed $\beta_{c}=100$. Data extracted from MPS simulations (yellow squares) is compared with the anayltical expression in the main text (blue dots). 


\section{Discussion}

The results presented in Section 2 demonstrate that accurate reduced system behaviour in the spin-boson model can be obtained in the presence of both a single or two finite-temperature environments. Non-perturbative effects related to system-bath entanglement (polaronic dresing) are captured in transient relaxation and non-equilibrium steady states, and we have shown how the T-TEDOPA transformation can be inverted to obtain direct information related to the energy and entanglement entropy flows in the environmental modes. All of these reults were obtained from pure wave function evolution of an initial zero-temperature (vacuum) state, and the onerous numerical cost of having to sample over a thermal distriubtion of intial states was entirely avoided.

However, we did note that the numerical resources required to obtain these results grew in an unbounded way as a function of simulation time. In the case of the one-bath SBM, Figure 6 shows the total number of bosonic excitation grows approximately linearly in time and the growth rate increases with the bath temperature. The main panel showing the populations of the environment in frequency space shows that this growth is the result of growing populations at frequencies $\approx \pm \omega_{0}$. In Section 2.2 we made the observation that creating an excitation in a negative frequency mode allows the TLS to be excited with overall conversation of energy, and this is the process that accounts for the 'heating' expected of a finite-temperature bath. The constant growth of excitations in the environment can be seen to arise from the constant cycling of the heating process sketched in Figure 12 ( a similar cycle for cooling also generates a net population of excitations). Here the the creation of a negative frequency excitation ( or hole) excites the TLS and then is de-excited by the creation of an excitation in the positive frequency environment.

Interestingly, this pair creation goes beyond populations: the dynamics of thermailsation entangles the positive and negative frequency environments, as seen in our Figure 7 . This is perhaps unsurprising in the context of the the thermofield theory of De Vega et al. where the thermal entanglement properties of two-mode squeezed states are used to create an effective finite temperature environment from two zero-temperature baths [56]. Another, hopefully fruitful, connection can be made to very recent developments in the theory of MPS and tensor networks for quantum transport. Non-equilibrium particle flows between reservoirs at different chemical potentials also lead to the constant creation of entangled particle-hole pairs, leading to the exponential-in-time growth of MPS bond dimensions. However, Rams and Zwolak have recently demonstrated that a change in basis used for certain fermionic transport simulations can greatly mitigate this rapid growth of numerical resources [57], and it would be very interesting to see how this might translate - or might to some extent already be implemented - in our current approach to bosonic heat flow problems. Finally, we also point out that that rapid growth of bond dimensions and entanglement in non-equilibrium systems is potentially a problem for 1TDVP simulations, as these proceed at fixed bond-dimensions. Choosing large bond-dimensions may allow one to reach long times, but much of the simulation is likely to run slowly, as it will be using far more resources than are necessary for most of the time. In a recent development, Dunnet and Chin have proposed an adaptive version of 1TDVP that is able to change bond-dimensions during the course of a single simulation run, allowing the necessary resources to be deployed as needed. 


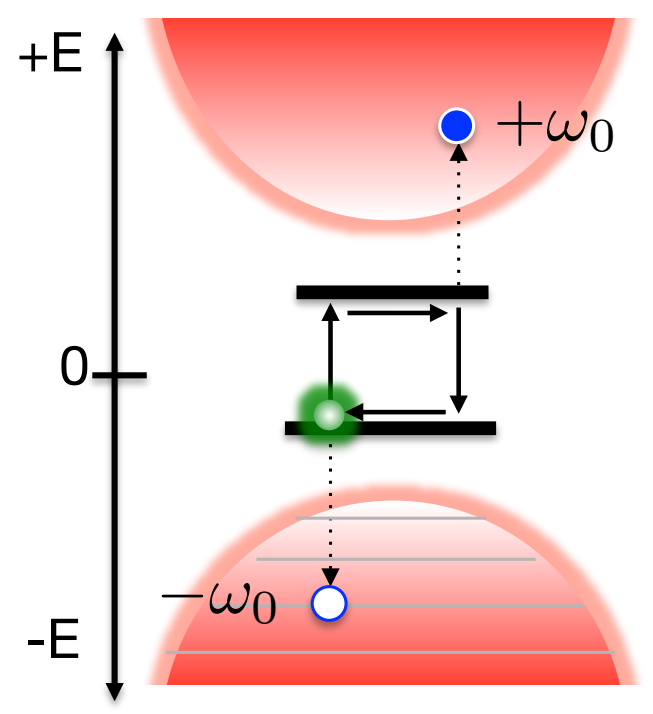

Figure 12. Due to the unbounded bosonic nature of the negative-frequency environment, thermal transitions within the TLS lead to a constant creation of correlated, particle-hole-like excitations in both environments with the same absolute energy $\omega_{0}$.

\section{Materials and Methods}

All numerical results have been obtained using software packages that are available at https://github.com/angusdunnett/MPSDynamics.

Author Contributions: Conceptualization, AWC; methodology, AJD \& AWC; software, AJD; writing-original draft preparation, AWC \& AJD; All authors have read and agreed to the published version of the manuscript.

Funding: ADJ Acknowledges support from Ecole Doctorale Physique en Ile-de-France (EDPIF ED564. AWC is partly supported by ANR project No. 195608/ACCEPT.

Conflicts of Interest: The authors declare no conflict of interest.

\section{References}

1. Weiss, U. Quantum Dissipative Systems, 4 ed.; WORLD SCIENTIFIC, 2012. doi:10.1142/8334.

2. Breuer, H.P.; Petruccione, F.; others. The theory of open quantum systems; Oxford University Press on Demand, 2002.

3. Acín, A.; Bloch, I.; Buhrman, H.; Calarco, T.; Eichler, C.; Eisert, J.; Esteve, D.; Gisin, N.; Glaser, S.J.; Jelezko, F.; others. The quantum technologies roadmap: a European community view. New Journal of Physics 2018, 20, 080201.

4. Gemmer, J.; Michel, M.; Mahler, G. Quantum thermodynamics: Emergence of thermodynamic behavior within composite quantum systems; Vol. 784, Springer, 2009.

5. Kosloff, R.; Levy, A. Quantum Heat Engines and Refrigerators: Continuous Devices. Annual Review of Physical Chemistry 2014, 65, 365-393. doi:10.1146/annurev-physchem-040513-103724.

6. Benenti, G.; Casati, G.; Saito, K.; Whitney, R. Fundamental aspects of steady-state conversion of heat to work at the nanoscale. Physics Reports 2017, 694, 1-124. doi:10.1016/j.physrep.2017.05.008.

7. Elenewski, J.E.; Gruss, D.; Zwolak, M. Communication: Master equations for electron transport: The limits of the Markovian limit. The Journal of chemical physics 2017, 147, 151101.

8. Thoss, M.; Evers, F. Perspective: Theory of quantum transport in molecular junctions. The Journal of chemical physics 2018, 148, 030901. 
9. Ishizaki, A.; Fleming, G.R. Unified treatment of quantum coherent and incoherent hopping dynamics in electronic energy transfer: Reduced hierarchy equation approach. The Journal of chemical physics 2009, 130, 234111.

10. Chin, A.; Prior, J.; Rosenbach, R.; Caycedo-Soler, F.; Huelga, S.F.; Plenio, M.B. The role of non-equilibrium vibrational structures in electronic coherence and recoherence in pigment-protein complexes. Nature Physics 2013, 9, 113-118.

11. Smith, S.L.; Chin, A.W. Ultrafast charge separation and nongeminate electron-hole recombination in organic photovoltaics. Physical Chemistry Chemical Physics 2014, 16, 20305-20309.

12. Oviedo-Casado, S.; Prior, J.; Chin, A.; Rosenbach, R.; Huelga, S.; Plenio, M. Phase-dependent exciton transport and energy harvesting from thermal environments. Physical Review A 2016, 93, 020102.

13. Chin, A.; Mangaud, E.; Atabek, O.; Desouter-Lecomte, M. Coherent quantum dynamics launched by incoherent relaxation in a quantum circuit simulator of a light-harvesting complex. Physical Review A 2018, 97, 063823.

14. Schröder, F.A.; Turban, D.H.; Musser, A.J.; Hine, N.D.; Chin, A.W. Tensor network simulation of multi-environmental open quantum dynamics via machine learning and entanglement renormalisation. Nature communications 2019, 10,1-10.

15. Maguire, H.; Iles-Smith, J.; Nazir, A. Environmental nonadditivity and franck-condon physics in nonequilibrium quantum systems. Physical review letters 2019, 123, 093601.

16. Wertnik, M.; Chin, A.; Nori, F.; Lambert, N. Optimizing co-operative multi-environment dynamics in a dark-state-enhanced photosynthetic heat engine. The Journal of chemical physics 2018, 149, 084112.

17. Del Pino, J.; Schröder, F.A.; Chin, A.W.; Feist, J.; Garcia-Vidal, F.J. Tensor network simulation of polaron-polaritons in organic microcavities. Physical Review B 2018, 98, 165416.

18. Weiss, U. Quantum dissipative systems; Vol. 13, World scientific, 2012.

19. Strathearn, A.; Kirton, P.; Kilda, D.; Keeling, J.; Lovett, B.W. Efficient non-Markovian quantum dynamics using time-evolving matrix product operators. Nature Communications 2018, 9, 3322. doi:10.1038/s41467-018-05617-3.

20. Topaler, M.; Makri, N. Quantum rates for a double well coupled to a dissipative bath: Accurate path integral results and comparison with approximate theories. The Journal of chemical physics 1994, 101, 7500-7519.

21. Prior, J.; Chin, A.W.; Huelga, S.F.; Plenio, M.B. Efficient simulation of strong system-environment interactions. Physical review letters 2010, 105, 050404.

22. Oviedo-Casado, S.; Prior, J.; Chin, A.W.; Rosenbach, R.; Huelga, S.F.; Plenio, M.B. Phase-dependent exciton transport and energy harvesting from thermal environments. Phys. Rev. A 2016, 93, 020102. doi:10.1103/PhysRevA.93.020102.

23. Somoza, A.D.; Marty, O.; Lim, J.; Huelga, S.F.; Plenio, M.B. Dissipation-Assisted Matrix Product Factorization. Phys. Rev. Lett. 2019, 123, 100502. doi:10.1103/PhysRevLett.123.100502.

24. Lindner, C.J.; Kugler, F.B.; Meden, V.; Schoeller, H. Renormalization group transport theory for open quantum systems: Charge fluctuations in multilevel quantum dots in and out of equilibrium. Physical Review B 2019, 99, 205142.

25. Wang, H.; Shao, J. Quantum Phase Transition in the Spin-Boson Model: A Multilayer Multiconfiguration Time-Dependent Hartree Study. The Journal of Physical Chemistry A 2019, 123, 1882-1893.

26. Haegeman, J.; Lubich, C.; Oseledets, I.; Vandereycken, B.; Verstraete, F. Unifying time evolution and optimization with matrix product states. Physical Review B 2016, 94, 165116. arXiv: 1408.5056, doi:10.1103/PhysRevB.94.165116.

27. Haegeman, J.; Cirac, J.I.; Osborne, T.J.; Pižorn, I.; Verschelde, H.; Verstraete, F. Time-Dependent Variational Principle for Quantum Lattices. Physical Review Letters 2011, 107, 070601. doi:10.1103/PhysRevLett.107.070601.

28. Schröder, F.A.Y.N.; Chin, A.W. Simulating open quantum dynamics with time-dependent variational matrix product states: Towards microscopic correlation of environment dynamics and reduced system evolution. Phys. Rev. B 2016, 93, 075105. doi:10.1103/PhysRevB.93.075105.

29. Gonzalez-Ballestero, C.; Schröder, F.A.Y.N.; Chin, A.W. Uncovering nonperturbative dynamics of the biased sub-Ohmic spin-boson model with variational matrix product states. Phys. Rev. B 2017, 96, 115427. doi:10.1103/PhysRevB.96.115427. 
30. Tamascelli, D.; Smirne, A.; Lim, J.; Huelga, S.F.; Plenio, M.B. Efficient simulation of finite-temperature open quantum systems. Physical Review Letters 2019, 123, 090402. arXiv: 1811.12418, doi:10.1103/PhysRevLett.123.090402.

31. Tamascelli, D.; Smirne, A.; Huelga, S.; Plenio, M. Nonperturbative Treatment of non-Markovian Dynamics of Open Quantum Systems. Physical Review Letters 2018, 120, 030402. doi:10.1103/PhysRevLett.120.030402.

32. Wilhelm, F.; Kleff, S.; Von Delft, J. The spin-boson model with a structured environment: a comparison of approaches. Chemical physics 2004, 296, 345-353.

33. Schulze, J.; Kuhn, O. Explicit correlated exciton-vibrational dynamics of the FMO complex. The Journal of Physical Chemistry B 2015, 119, 6211-6216.

34. Mendive-Tapia, D.; Mangaud, E.; Firmino, T.; de la Lande, A.; Desouter-Lecomte, M.; Meyer, H.D.; Gatti, F. Multidimensional quantum mechanical modeling of electron transfer and electronic coherence in plant cryptochromes: The role of initial bath conditions. The Journal of Physical Chemistry B 2018, 122, 126-136.

35. May, V.; Kühn, O. Charge and energy transfer dynamics in molecular systems; John Wiley \& Sons, 2008.

36. Mukamel, S. Principles of nonlinear optical spectroscopy; Vol. 6, Oxford university press New York, 1995.

37. Alvermann, A.; Fehske, H. Sparse polynomial space approach to dissipative quantum systems: Application to the sub-ohmic spin-boson model. Physical review letters 2009, 102, 150601.

38. Binder, R.; Burghardt, I. First-principles quantum simulations of exciton diffusion on a minimal oligothiophene chain at finite temperature. Faraday Discussions 2019, 221, 406-427.

39. Jiang, T.; Li, W.; Ren, J.; Shuai, Z. Finite Temperature Dynamical Density Matrix Renormalization Group for Spectroscopy in Frequency Domain. The Journal of Physical Chemistry Letters 2020, 11, 3761-3768. PMID: 32316732, doi:10.1021/acs.jpclett.0c00905.

40. Tamascelli, D.; Smirne, A.; Huelga, S.F.; Plenio, M.B. Nonperturbative treatment of non-Markovian dynamics of open quantum systems. Physical review letters 2018, 120, 030402.

41. Musser, A.J.; Liebel, M.; Schnedermann, C.; Wende, T.; Kehoe, T.B.; Rao, A.; Kukura, P. Evidence for conical intersection dynamics mediating ultrafast singlet exciton fission. Nature Physics 2015, 11, 352-357.

42. Schnedermann, C.; Lim, J.M.; Wende, T.; Duarte, A.S.; Ni, L.; Gu, Q.; Sadhanala, A.; Rao, A.; Kukura, P. Sub-10 fs time-resolved vibronic optical microscopy. The journal of physical chemistry letters 2016, 7, 4854-4859.

43. Schnedermann, C.; Alvertis, A.M.; Wende, T.; Lukman, S.; Feng, J.; Schröder, F.A.; Turban, D.H.; Wu, J.; Hine, N.D.; Greenham, N.C.; others. A molecular movie of ultrafast singlet fission. Nature communications 2019, 10, 1-11.

44. Chin, A.W.; Rivas, Á.; Huelga, S.F.; Plenio, M.B. Exact mapping between system-reservoir quantum models and semi-infinite discrete chains using orthogonal polynomials. Journal of Mathematical Physics 2010, 51, 092109.

45. Prior, J.; de Vega, I.; Chin, A.W.; Huelga, S.F.; Plenio, M.B. Quantum dynamics in photonic crystals. Physical Review A 2013, 87, 013428. arXiv: 1205.2897, doi:10.1103/PhysRevA.87.013428.

46. Chin, A.W.; Rivas, A.; Huelga, S.F.; Plenio, M.B. Exact mapping between system-reservoir quantum models and semi-infinite discrete chains using orthogonal polynomials. Journal of Mathematical Physics 2010, 51, 092109. arXiv: 1006.4507, doi:10.1063/1.3490188.

47. Silbey, R.; Harris, R.A. Variational calculation of the dynamics of a two level system interacting with a bath. The Journal of Chemical Physics 1984, 80, 2615-2617. doi:10.1063/1.447055.

48. Dubi, Y.; DiaVentra, M. Colloquium: Heat flow and thermoelectricity in atomic and molecular junctions. Reviews of Modern Physics 2011, 83, 131-155. doi:10.1103/RevModPhys.83.131.

49. Dhar, A. Heat transport in low-dimensional systems. Advances in Physics 2008, 57, $457-537$. doi:10.1080/00018730802538522.

50. Guo, C.; Weichselbaum, A.; vonDelft, J.; Vojta, M. Critical and Strong-Coupling Phases in One- and Two-Bath Spin-Boson Models. Physical Review Letters 2012, 108, 160401. doi:10.1103/PhysRevLett.108.160401.

51. Zhou, N.; Chen, L.; Xu, D.; Chernyak, V.; Zhao, Y. Symmetry and the critical phase of the two-bath spin-boson model: Ground-state properties. Physical Review B 2015, 91, 195129. doi:10.1103/PhysRevB.91.195129.

52. Bruognolo, B.; Weichselbaum, A.; Guo, C.; von Delft, J.; Schneider, I.; Vojta, M. Two-bath spin-boson model: Phase diagram and critical properties. Physical Review B 2014, 90, 245130. doi:10.1103/PhysRevB.90.245130. 
53. Segal, D.; Nitzan, A. Spin-Boson Thermal Rectifier. Physical Review Letters 2005, 94, 034301. doi:10.1103/PhysRevLett.94.034301.

54. Chen, T.; Balachandran, V.; Guo, C.; Poletti, D. Steady state quantum transport through an anharmonic oscillator strongly coupled to two heat reservoirs. arXiv:2004.05017 [cond-mat] 2020. arXiv: 2004.05017.

55. Blunden-Codd, Z.; Bera, S.; Bruognolo, B.; Linden, N.O.; Chin, A.W.; Von Delft, J.; Nazir, A.; Florens, S. Anatomy of quantum critical wave functions in dissipative impurity problems. Physical Review B 2017, 95, 085104.

56. de Vega, I.; Bañuls, M.C. Thermofield-based chain-mapping approach for open quantum systems. Physical Review A 2015, 92, 052116. doi:10.1103/PhysRevA.92.052116.

57. Rams, M.M.; Zwolak, M. Breaking the Entanglement Barrier: Tensor Network Simulation of Quantum Transport. Phys. Rev. Lett. 2020, 124, 137701. doi:10.1103/PhysRevLett.124.137701. 